\title{
Segmentation of the Subthalamic Nucleus in MR Images Using Information Fusion - A Preliminary Study for a Computed-Aided Surgery of Parkinson's Disease
}

\author{
Vincent Barra $^{1}$, Jean-Jacques Lemaire ${ }^{1,2}$, Franck Durif ${ }^{3}$, Jean-Yves Boire ${ }^{1}$ \\ ${ }^{1}$ ERIM, ${ }^{2}$ URN, ${ }^{3}$ Department of Neurology, Faculty of Medicine, BP 3863001 \\ CLERMONT-FERRAND, France \\ \{vincent.barra, j-yves.boire\}@u-clermont1.fr
}

\begin{abstract}
Subthalamic nucleus (ST) stimulation is proved to have beneficial effects on the symptoms of Parkinson's disease. We propose a fully automated segmentation method of this structure on MR images based on an information fusion technique. Information is provided by both images and expert knowledge, and consists in morphological, topological and tissue constitution data. All this ambiguous, complementary and redundant information is managed using a three steps fusion scheme based on fuzzy logic. Information is first modeled into a common theoretical frame managing imprecision and uncertainty. Models are then fused and a segmentation is finally performed that reduce the imprecision and increase the certainty in the location of the structure. Computed locations are compared with those obtained during a stereotactic surgical procedure. Results on ten patients are very promising, and suggest that this method may be applied during the surgical procedure as an help for the location of the subthalamic nucleus.
\end{abstract}

\section{Method}

In order to segment the ST, we were first interested in locating the third ventricle (V3) and the two red nuclei (RN). V3 was defined as a "cerebrospinal fluid (CSF) area, roughly located in the inter hemispheric plane, at approximately $20 \mathrm{~mm}$ under the lateral ventricles". RN as for them were defined as "ovoid-shape structures, almost symmetrical with respect to the inter hemispheric plane, approximately $7 \mathrm{~mm}$ long in the left-right axis, and $9 \mathrm{~mm}$ long in the antero-posterior axis, with a white matter-like (WM) signal in T2-weighted images". Finally, the ST were defined by the neurosurgeon as "gray matter (GM) structure, almost symmetrical with respect to the inter hemispheric plane, $15 \mathrm{~mm}$ under or $10 \mathrm{~mm}$ under and anterior the $\mathrm{RN}$, posterior and inferior to the V3 (at a distance of almost $8 \mathrm{~mm}$ )". We then defined the following scenario in order to precisely locate the ST:

- $\quad$ segmentation of the ventricular system and V3 using topological information ;

- $\quad$ segmentation of the RN from the V3 using topological and tissue information

- $\quad$ segmentation of the STN from the RN using topological, and tissue information 
All the ambiguous information was modeled in a fuzzy logic frame, allowing the management of imprecision and incertitude inherent to the medical data. More precisely, each piece of information was theoretically modeled as a fuzzy map, where the gray level of a voxel represented its membership to the studied information (tissue constitution, distance, direction). All these fuzzy maps were then aggregated according to their redundancy and their complementarities, using concepts derived from data fusion. Finally a decision (i.e. a segmentation of ST) was processed following the aggregation step, and results of this segmentation were compared to those obtained during a stereotactic surgical procedure. All the interest of data fusion here was that decision was taken by managing all the available information. Moreover, further data (morphological aspect of the ST for example) might simply be added to the process.

\section{Results and Conclusion}

The following figure presents an overview of the process. Points of the ST were represented in black in the last image

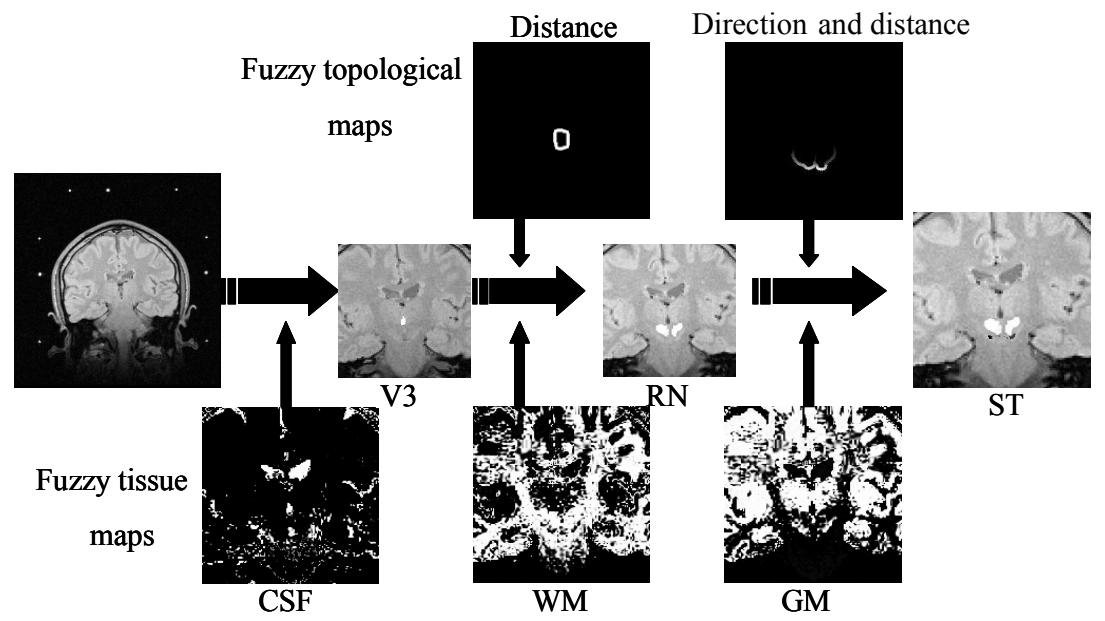

Ten patients were selected for electrical stimulation of ST. Three orthogonal MR images were acquired in stereotactic conditions (Leksell Model $G$ frame and four unicortical fixations repositioning system, Elekta Instruments ${ }^{\circledR}$ ) on a Magnetom $1 T$ (Siemens). Stereotactic coordinates of the center of inertia of the computed ST were compared for the ten patients to stereotactic coordinates of the point considered as the most efficient for stimulation by neurosurgeons after the surgical procedure. Results of this preliminary study were quite encouraging, but still perfectible, and we now plan to improve the results by taking into account the real 3D shape of the ST using the three orthogonal MR sequences provided by the protocol. 